\title{
Early Detection of Microcalcification in Digital Mammograms using Neural Network
}

\author{
S. Sakthi ${ }^{1}$, M. Kumar ${ }^{2}$ \\ Asst Professor, Information Technology, Chettinad College of Engineering and Technology, Karur, Tamilnadu, India ${ }^{1,2}$
}

\begin{abstract}
The most common and first method used to detect breast lesions is mammography. Due to less visibility, poor contrast and noisy nature of mammogram it is necessary to detect the small and non-noticeable cancers in early stage. In order to interpret the changes caused due to mental disorder of the breast the contrast of the images are improved while screening the mammograms. The architectural distortion is found in interpreting breast cancers as well as microcalcification and mass on mammograms. However, it is more difficult to detect architectural distortion than microcalcification and mass. The original mammogram image is decomposed using wavelet decomposition and gabor features are extracted from the original image Region of Interest (ROI). The ability of these features in detecting microcalcification is done using Backpropagation Neural Network (BPNN). The proposed classification approach is applied to a database of 322 dense mammographic images, originating from the MIAS database.This paper presents various techniques used for automatic enhancement and segmentation of microcalcifications in mammographic images. These techniques consists of three different stages which includes preprocessing stage, feature extraction stage and classification stage. The paper represents the proposed system in which these stages can be implemented using histogram equalization, contrast stretching and image segmentation.
\end{abstract}

Keywords: Mammography, Microcalcifications, Preprocessing, Feature Extraction, Classification, Histogram Equalization, Contrast Stretching, Backpropagation Neural Network and Image Segmentation.

\section{INTRODUCTION}

Breast cancer is a type of cancer which is generated from Microcalcifications (MC) are quiet tiny bits of calcium, breast tissue. Breast cancer occurs in humans and other and may show up in clusters or in patterns and are mammals while the majority of human cases are in associated with extra cell activity in breast tissue. Usually women. Breast cancer is a type of malignant tumor that the extra cell growth is not cancerous, but sometimes tight begins in the cells of the breast. A malignant tumor is a clusters of microcalcification can indicate early breast collection of cancer cells that can grow into surrounding cancer. Scattered microcalcifications are usually a sign of tissues or spread to different areas of the body. The disease benign breast cancer. $80 \%$ of the MC is benign. MC in the occurs almost entirely in women. As compared to breast shows up as white speckles on breast X-rays. The developing nation Breast cancer is highly occurred in calcifications are small; usually varying from 100 developed ones. The major reason behind this is the micrometer to 300 micrometer, but in reality may be as amount of time one is expected to live. Due to different large as $2 \mathrm{~mm}$. Though it is very difficult to detect the lifestyles and eating habits of females in rich and poor calcifications as such, when more than 10 calcifications countries breast cancer is mostly occurred in elderly are clustered together, it becomes possible to diagnose women because women in the richest countries is expected malignant disease. But the survival depends on how early to live for longer duration than the women in poor country. the cancer is detected. So, any MC formation should be Breast cancer is the major cause of cancer death among detected at the benign stage. Hence, a Computer Aided women. The most secure detection of early and curable Diagnosis (CAD) system is used to detect MC clusters. breast cancer is mammography. Early detection of breast Classification of microcalcifications in digital cancer is more efficient because mammography is the mammogram consists of three main stages preprocessing most standard exam to first detect breast cancer. stage, feature extraction stage and classification stage.

Mammography is the method which can be used to detect The main advantage of Digital mammography is the different infections that are breast cancer characteristics storage of mammogram images digitally and thereby such as microcalcifications. In western countries about reducing the no of patient biopsies. The image 53\%-92\% of the population has this disease. In a enhancement techniques applied to the digitally stored Philippines study a mammogram screening was done to images helps to get clearer and contrast enhanced images 151,198 women. Out of that 3479 women had this disease which majorly helps the radiologists more than screen film and were referred for diagnosis. Though breast cancer mammography in diagnosing the patients.

leads to death, early detection of breast cancer can In Digital mammography four important image processing increase the survival rate. The current diagnostic method techniques are used which all together helps the for early detection of breast cancer digital mammography. radiologists to give accurate results of the diagnosis. The 
Vol. 5, Issue 12, December 2016

techniques used are- preprocessing, segmentation, feature extraction and classification. In this paper all these techniques are discussed by taking different methods for each of them. The block diagram in figure 2 represents the flow of the process. The final output will be the mass detected as either benign or malignant tumor.

\section{Preprocessing of Digital Mammograms:}

In the preprocessing stage the mammogram image is improved by enhancing the features of the mammogram image. In the step all the unnecessary distortion are removed from the image. In preprocessing stage the contrast of the image is enhanced and the noise from the image is removed improving and enhancing the image data. Due to this step image data is improved and ready for feature extraction stage. The proposed algorithm helps radiologist to diagnosis the disease more accurately and takes decision swiftly. The types of noise observed in the mammogram image are marked in the Figure 1. In the proposed algorithm, it abolishes all these unwanted and surplus noises from the mammogram image.

The process begins by obtaining the digital mammogram images at different quantization and sampling rates. Denoising techniques are applied to remove the noise from the images and then contrast enhancement techniques are applied to enhance the mammogram images. At this level, several approaches have been adopted like wavelet transform, multi-resolution analysis, region growing techniques...etc. The following part highlights the different mammogram image de-noising and enhancement approaches.

\section{A. Mammogram image De-noising using wavelet multi- resolution technique. It has been revealed that, at the time of taking the breast images using X-rays, the radiation exposure caused by mammography exam can induce the development of breast cancer in some women undergoing the test. One solution to this problem is the use of reduced radiation doses. But, the use of such reduced doses of radiations increases the quantum noise in the mammogram images due to the use of low photon count for the image formation process. The presence of such quantum noise reduces the visibility of subtle lesions in the mammograms.}

To deal with this problem, new approach has been presented, using wavelet multi-resolution image analysis .In this approach the mammogram images are obtained at different radiation dose levels: $100 \%, 85 \%, 70 \%$ and $50 \%$ of the standard dose.

On these images the multi-resolution technique is applied. The steps are: i. Decompose the input signal with forward wavelet transform. ii. Apply a thresholding method to the vertical, horizontal and diagonal coefficients. iii. Apply the wavelet inverse transform in order to reconstruct the signal. The co-efficient shrinkage in wavelet domain is used with the multi-resolution technique to de-noise the images.
Pre-processing of mammography helps to identifies abnormal areas that cannot be experienced physically or visualized, but can be detected through CAD. Imaging techniques play an important role in CAD. The CAD helps doctors and radiologists to diagnose and identify the abnormality quickly and easily. Here, the proposed method helps standardize the image quality and extracting the target ROI. By breast part extraction, muscle part removal and enhancement of mammogram. The proposed algorithm helps radiologist to diagnosis the disease more accurately and takes decision swiftly. The types of noise observed in the mammogram image are marked in the Figure 1. In the proposed algorithm, it abolish all these unwanted and surplus noises from the mammogram image.

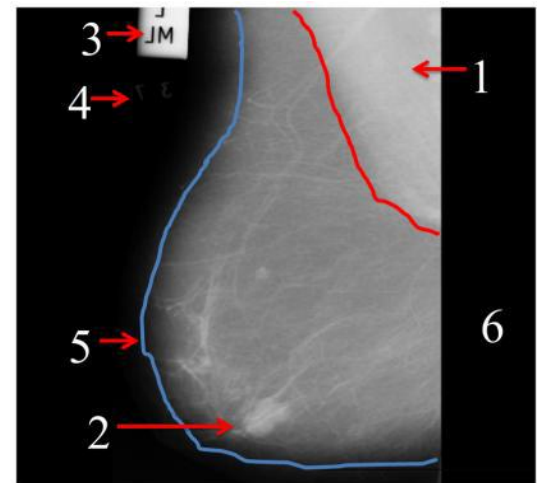

Fig 1 .Types of noises observed in original image and marked with numbers as 1 . Pictorial Muscle, 2.Tumor, 3.High Intensity, 4.Low Intensity, 5. Breast Part and 6. Background.

\section{Segmentation of Digital Mammograms:}

Segmentation is the process of dividing the image into its constituent parts or objects from which the regions of interest are selected. It is the second main stage of Digital mammography, in which the suspicious regions that may contain the masses are separated from the background parenchyma tissue. The separation done in this way divides the mammogram image into several nonoverlapping regions from which the Region of Interest (ROI) are extracted. From the selected ROI the suspicious mass candidates are located. Usually the brightness of the suspicious areas is higher than its surroundings and also they almost have uniform density, regular shape with varying size and fuzzy boundaries. Since the mass characteristics vary from one image to the other image, the segmentation of masses is a complex process. The following discussion covers some of the approaches for the mammogram image segmentation. The approaches include digital image processing techniques like median filtering, texture analysis, nearest neighboring algorithm...etc.

A. Segmentation of breast masses in Digital mammograms using Adaptive median filtering and texture analysis. In this approach, adaptive median filtering and texture analysis are used to divide the 
Vol. 5, Issue 12, December 2016

mammogram image into multiple segments in order to denoising and contrast enhancement. However, it is identify the Region of Interest (ROI) consisting of important to enhance the contrast and denoise the image at potential masses. There are three steps involved in this the same time. In order to increase the accuracy, a good approach: i. Small neighborhood removal - the test images image enhancement should enhance the contrast of after preprocessing consists of some small neighborhood mammogram images and remove or decrease the image pixels that create negative impact on the segmentation. noise simultaneously.

Since these neighborhoods are not bright enough to be considered as potential masses, they must be removed first.

\section{Feature Extraction of Digital Mammograms:}

Feature extraction is an essential step required for pattern recognition. Feature extraction is used to extract features from a large amount of data. In other words it simplifies the amount of features required to describe the large set of data accurately. The features which represent important information for the classification are defined using feature extraction process. Feature extraction is a process which is used for identification of target data by extracting spatial data such as texture,size,etc. It is also used to restructure the data to improve the performance and remove the image data that is redundant or of very less value in the classification process. Hence in this way it is used for pattern recognization.

\section{A. Feature Extraction by calculating the Roughness} value. In this work, a mammogram image is used for the breast cancer detection. The roughness of each and every pixel is calculated. The use of fractal analysis reduces the search region. If the roughness value calculated for a region lies between 2 to 3 , then the region is considered to be having a potential mass. All the other sub-blocks with roughness value less than 2 or greater than 3 have to be discarded since they are not considered as masses. The various features selected in this approach to locate the regions that are suspicious of tumors are: area of the surface, compactness, mean gradient within current region, mean gradient of region boundary, gray value variance.

\section{Classification of Digital Mammograms:}

Classification is a process which is used to classify the features extracted in the feature extraction stage. This is one of the main process that is used for identification of data extracted with the target data. In mammogram images first the mammograms are classified into normal and abnormal. If the image consists of microcalcifications then it is classified as abnormal image. In the next step the abnormal mammogram is further classified into malignant or benign. There are various methods used for classification of microcalcifications in digital mammogram such wavelet transform, contourlet transform, etc.

\section{RELATED WORK}

In order to improve the quality of mammogram images many image enhancement techniques have been developed in this recent years. Enhancement refers to improving the contrast of the mammogram images by achieving

\section{PROPOSED SYSTEM}

The mammogram images needs to be improved as it is very difficult to classify the various disorders within that image due to the less visibility of the image. The proposed system uses the modified non subsampled contourlet transform (NSCT) to classify the various microcalcifications within the digital mammogram images.

The proposed system can be used for performing enhancement of the image, segmentation of the disorders present within the image as well as classification of the various disorders present in the image. Therefore nonsubsampled contourlet transform can prove to be one of the most manageable and effective technique to be used for classifying the microcalcifications with the mammogram image. Fig. 2 illustrates the overall working of the proposed system.

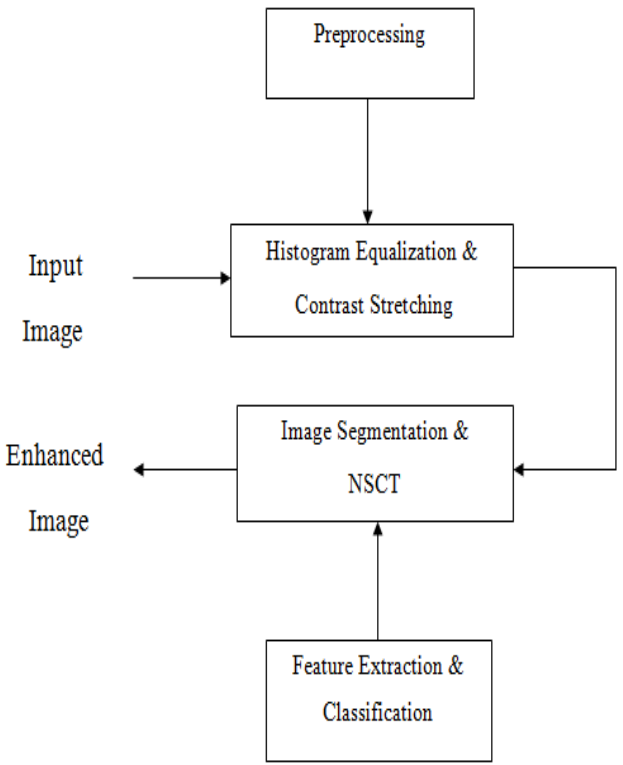

Fig. 2 Overall process of the proposed system

The main purpose of a breast cancer CAD system is to help the radiologist and doctors to take decision swiftly. By providing exact ROI will help to identify abnormality. The proposed method works in three stages as explained in figure 2. The first step is to remove the back ground artefacts (label 3,4 and 6) identified in the figure 1.The second step is to reduce the pectoral muscle (label 1) identified in figure 2 .

The overall process of the working of the proposed model consists of the following steps: 


\section{International Journal of Advanced Research in Computer and Communication Engineering} ISO 3297:2007 Certified

Vol. 5, Issue 12, December 2016

1. Histogram equalization and contrast stretching are the two steps that are used for preprocessing of the input mammogram image.

2. This steps are basically used to enhance the mammogram image by maximizing the information of the mammogram image by adjusting the gray levels of the input image and improving the visibility of the input image.

3. Nonsubsampled contourlet transform is the third step which is used for image segmentation. This step is used to perform feature extraction. The enhanced mammogram image is segmented for finding microcalcifications in it.

4. The area within the image that are found to be suspicious or faulty are segmented from the image and further classified for various disorders by decomposing the image into multiple scales and multiple directions.

5. In the last step the nonsubsampled contourlet
transform is used for classification of the segmented transform is used for classification of the segmented
suspicious areas into the various abnormalities such as benign or malignant tumors.

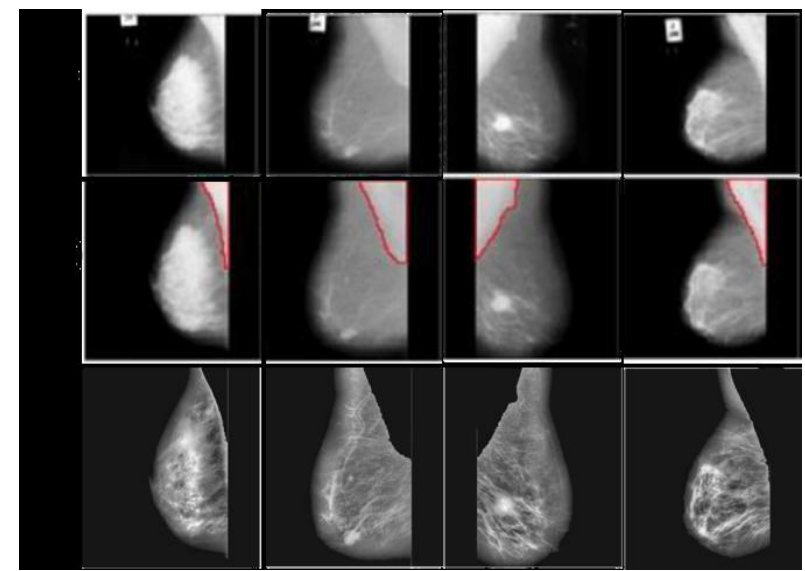

Fig 3. Comparison of result (a) Original Images (b) Farhan et al. results (c) Results of our proposed Method

Figure 3 illustrates the comparison results between existing work (fig3b) and proposed work (fig3c). These results suggest that, our current study has convincingly enhanced the quality of the image with better in contrast.

\section{CONCLUSION}

Breast cancer is leading cause of death for women. Timely screening may help to detect the abnormality but current screening method, the mammography is low dose x-ray image. It may miss the small changes in the breast because of low dose x-ray it generates poor contrast image. The missed changes may lead for serious problem.

Hence, CAD system helps to identify the diminutive changes in the breast. This early detection may save the life of the patient. The proposed method of pre-processing presented with removal of background artefacts, pectoral muscle suppression and image quality enrichment helps much in early detection. A variety of research has been occurred on mammography. There are lot of various techniques that are used for classification of microcalcifications in mammogram images. Each of these techniques have their own specific contribution and limitations.

In order to solve the various problems that occurs in these techniques a system can be proposed which solves this problems. This system can make use of nonsubsampled contourlet transform for the classification of microcalcifications in digital mammogram images for giving improved results.

We present a novel approach to the problem of computeraided analysis of digital mammograms for breast cancer detection. The algorithm developed here classifies mammograms into normal \& abnormal.

First, the structures in mammograms produced by normal glandular tissue of varying density are eliminated using a Integer Wavelet Transform (IWT) based local average subtraction. The gabor features are extracted and classification approaches using artificial neural networks shows good classification results. Using the mammographic data from the Mammographic Image Analysis Society (MIAS) database a recognition score of $84.3 \%$ was achieved using the proposed approach.

\section{REFERENCES}

[1] Laine, J. Fan and W. Yang, Wavelets for contrast enhancement of digital mammography, IEEE Engineering in Medicine and Biology, Sep./Oct. 1995, pp. 536-550.

[2] H.Wang, Y. Chen, T. Fang, J. Tyan and N. Ahuja, Gradient Adaptive Image Restoration and Enhancement, IEEE Proc. Int. Conf. Image Processing, Oct. 2006, pp. 2893-2896.

[3] R. C. Gonzalez and R. E. Woods, Digital Image Processing, 2nd edition Pearson Education, 2002.

[4] Y. Kopsinis and S. Mclaughlin, Development of EMD-Based Denoising Methods Inspired by Wavelet Thresholding, IEEE Trans. Signal Processing, Apr. 2009, vol.57, no.4, pp.1351- 1362.

[5] M. N. Do, and M. Vetterli., The countourlet transform: an efficient directional multiresolution image representation, IEEE Transactions on Image Processing, 2005, vol. 14, pp. 2091- 2106.

[6] D. D.-Y. Po and M. N. Do, Directional multiscale modeling of images using the contourlet transform, IEEE Trans. Image Proc., to appear, http://www.ifp.uiuc.edu/ $/$ minhdo/publications.

[7] S. Anand and R. Aynesh Vijaya Rathna, Detection of Architectural Distortion in Mammogram Images using Contourlet Transform, IEEE International Conference on Emerging Trends in Computing, Communication and Nanotechnology, 2013.

[8] Rekha Lakshmanan and Vinu Thomas, Enhancement of microcalcification features using morphology and contourlet Transform, IEEE International Conference on Advances in Computing and Communications, 2012.

[9] Sharanya Padmanabhan and Raji Sundararajan, Enhanced Accuracy of Breast Cancer Detection in Digital Mammograms using Wavelet Analysis, IEEE, 2012.

[10] K.Vaidehi, T.S.Subashini - "A global approach for detecting mass in Digital Mammograms" International Journal of Advancements in Research \& Technology, Volume 1, Issue6, November-2012 1 ISSN 2278-7763.

[11] Priyanka B Burshi, Digambar A Kulkarni, Digital Mammography: A Review on Detection of Breast Cancer, International Journal of Advanced Research in Computer and Communication Engineering Vol. 5, Issue 1, January 2016 


\section{BIOGRAPHY}

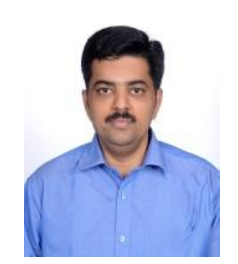

S. Sakthi, received the B.TECH degree in Information Technology from Adhiparasakthi Engineering College (Madras University) in the year 2002 and M.E degree in Computer Science and Engineering from Kongu Engineering College in the year 2005. He is currently working towards the Ph.D degree in the department of Information and Communication Engineering, Anna University, Chennai. His research interest includes Medical Image Processing, Image Processing and Artificial Intelligence.

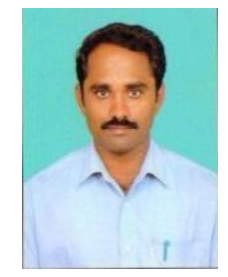

M. Kumar, received the B.E degree in Electronics and Communication Engineering from Bharathidasan University in the year 2003 and M.E degree in Applied Electronics from Sathyabama University in the year 2008. $\mathrm{He}$ is currently working towards the Ph.D degree in the department of Information and Communication Engineering, Anna University, Chennai. His research interest includes Speech signal processing, Image Processing and Embedded systems. 